\title{
Synergy of Paracrine Signaling During Early-Stage Mouse Ovarian Follicle Development In Vitro
}

\author{
Hong Zhou, ${ }^{1}$ Joseph T. Decker, ${ }^{1}$ Melissa M. Lemke, ${ }^{1}$ Claire E. Tomaszweski, ${ }^{1}$ Lonnie D. Shea, ${ }^{1,2}$ \\ Kelly B. Arnold, ${ }^{1}$ and Ariella Shikanov ${ }^{1,3,4}$ \\ ${ }^{1}$ Department of Biomedical Engineering, University of Michigan, Ann Arbor, USA; ${ }^{2}$ Department of Chemical Engineering, \\ University of Michigan, Ann Arbor, USA; ${ }^{3}$ Department of Macromolecular Science and Engineering, University of Michigan, \\ Ann Arbor, USA; and ${ }^{4} 2126$ Lurie Biomedical Engineering, 1101 Beal Avenue, Ann Arbor, MI 48109-2110, USA
}

(Received 14 February 2018; accepted 20 July 2018; published online 9 August 2018)

Associate Editor Michael R. King oversaw the review of this article.

\begin{abstract}
Introduction-Paracrine signals, such as soluble cytokines and extracellular matrix cues, are essential for the survival and development of multicellular ovarian follicles. While it is well established that hydrogel-based culture systems successfully support the growth of late-stage follicles for fertility preservation, growing small, early-stage ovarian follicles still proves to be challenging. We hypothesized that paracrine factors secreted from neighboring follicles may be crucial for improving the survival of early-stage follicles in vitro.

Methods - To test our hypothesis, we investigated the bidirectional crosstalk of the paracrine signals, such as cellsecreted cytokines, sex hormones and transcription factors (TFs), in follicles encapsulated and cultured for 12 days in alginate in groups of five $(5 x)$ and ten $(10 x)$.

Results-The differential profiles of TF activity and secretome during folliculogenesis were analyzed using TRanscriptional Activity CEllular aRray (TRACER) and data-driven
\end{abstract}

Address correspondence to Ariella Shikanov, 2126 Lurie Biomedical Engineering, 1101 Beal Avenue, Ann Arbor, MI 481092110, USA. Electronic mail: shikanov@umich.edu

Dr. Ariella Shikanov is an Assistant Professor in the Department of Biomedical Engineering at the University of Michigan, Ann Arbor. Her research aims to address the current limitations in treating and preventing premature ovarian failure and creating broad fertility preservation options for young cancer survivors. To achieve this, they create artificial constructs of ovarian tissue that direct tissue regeneration and restore biological function by combining approaches from engineering, materials, chemistry and life sciences. Dr. Shikanov has received numerous awards for her excellence in research, teaching and outreach activities, including The Harwell Foundation award (2014), National Science Foundation CAREER award (2016), Outstanding Undergraduate Research Opportunity Program Mentor (2014) and nominations for the Golden Apple Teaching award (2017 and 2018). Dr. Shikanov received her Bachelor's and PhD in Pharmaceutical Sciences and Medicinal Chemistry from the Hebrew University in Jerusalem from Prof. Abraham Domb, and completed her postdoctoral fellowship at Northwestern University in Chicago with Profs. Lonnie Shea and Teresa Woodruff in a multidisciplinary collaboration called the Oncofertility Consortium, which was created to address infertility induced by chemotherapy in cancer survivors. issue.

This article is part of the 2018 CMBE Young Innovators special multivariate modeling approach. The mechano- and oxygenresponsive TFs, NF- $\kappa \mathrm{B}$ and $\mathrm{HIF} 1$, exhibited a unique upregulation signature in $10 \times$ follicles. Consistently, levels of proangiogenic factors, such as VEGF-A and angiopoietin2 , were significantly higher in $10 \times$ follicles than those in $5 \times$ follicles, reaching 269.77 and $242.82 \mathrm{pg} / \mathrm{mL}$ on the last day of culture. The analysis of TRACER and secreted cytokines also revealed critical early interactions between cytokines and TFs, correlating with the observed phenotypical and functional differences between conditions.

Conclusions - We identified unique signatures of synergism during successful early-stage ovarian follicle development. These findings bring us closer to understanding of mechanisms underlying the downstream effects of interactions between the extracellular microenvironment and early-stage folliculogenesis in vitro.

Keywords-Primary ovarian follicle, Synergy, Paracrine signaling.

\section{INTRODUCTION}

Development of an ovarian follicle, a multicellular functional and structural unit of the ovary, depends on coordination between intrafollicular components, such

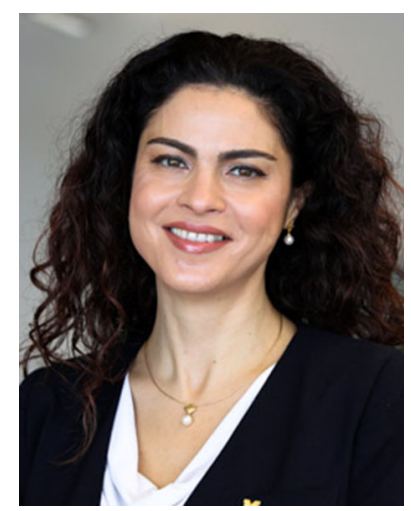


as the germ and stromal cells, and the extrafollicular ovarian components, such as stromal cells, ${ }^{61}$ macrophages ${ }^{65}$ and neighboring follicles. ${ }^{26}$ It is well established that hydrogel-based encapsulating culture systems can successfully support the growth of latestage ovarian follicles. However, the more abundant but under-utilized early-stage follicles are difficult to grow in vitro. A plausible explanation for the inability to grow individual small ovarian follicles in vitro is that early-stage follicle growth is largely gonadotropin-independent and rather under the control of paracrine and autocrine signals from the native ovarian environment, ${ }^{35}$ from which the follicles are deprived during the isolation process.

Paracrine and autocrine signals, such as soluble cytokines and extracellular matrix cues, are essential for the survival and development of primary follicles. $^{26,61,65}$ Recreation of these signals in vitro can mimic the native stromal support for small primary follicles with a stage-specific dependence on the ovarian environment. ${ }^{35}$ For example, isolated primary follicles can survive and mature when cultured in the presence of purified ovarian stroma composed of theca-interstitial cells and macrophages ${ }^{65}$ or mouse embryonic fibroblasts (MEFs) as a feeder cell layer. ${ }^{61}$ It has also been shown that culturing primary follicles in groups of ten, but not five, improved their survival and growth, ${ }^{26}$ while co-culturing with denuded oocytes, oocyte-secreted factors, or granulosa cells alone were insufficient to support early follicle growth in vitro. ${ }^{26}$ Altogether, these findings suggest that a bidirectional crosstalk between follicles via paracrine signals is central for successful folliculogenesis in vitro. Yet, the identity, concentrations and temporal secretion patterns of these signals remain unknown. The knowledge of these parameters could elucidate the specific roles of the factors in the paracrine signaling, and reveal the mechanisms that determine the success of folliculogenesis in vitro.

Capturing the dynamic changes occurring during follicle development requires experimental methods that give a systems-level view of factors' activity in a quantitative manner. In this study, we utilized encapsulated in vitro follicle growth systems (eIVFGs) that support folliculogenesis. ${ }^{53-55,74-76} \mathrm{We}$ hypothesized that coordinated activation of factors responsible for cell proliferation, follicle expansion, activation of hormone synthesizing enzymes and formation of centrally located, fluid-filled cavity, called antrum, creates a unique signature in the favorable condition where multiple (10 or more) co-cultured follicles survive and mature, compared to the incompetent condition where follicles ( 5 or less) arrest in their development. Herein, we described an integrated, systems-level approach to quantitatively characterize the real-time dynamic quantification of TF activity combined with cytokine and hormone profiles in primary mouse ovarian follicles cultured in alginate hydrogels in groups of $5(5 \times)$ or $10(10 \times)$ follicles.

To investigate the dynamic activity of TFs in growing follicles over the 12-day culture period, we employed TRanscriptional Activity CEllular aRray (TRACER). ${ }^{3,16,50,71}$ In transduced primary follicles, we established the expression of four TFs (p53, NF$\kappa \mathrm{B}$, Gli, and HIF1) associated with follicle development at various stages of folliculogenesis. First, p53 is a cell cycle regulator that fluctuates when cells proliferate. We hypothesized that granulosa cell proliferation, which is associated with follicle growth, would be reflected in $\mathrm{p} 53$ activities. Second, NF- $\kappa \mathrm{B}$ is responsive to substrate stiffness, ${ }^{23,29}$ and an anti-apoptotic factor. ${ }^{13}$ We hypothesized that greater upregulation of $\mathrm{NF}-\kappa \mathrm{B}$ in the $10 \times$ condition would correspond with the survival of granulosa cells and temporally correlate with follicle expansion. ${ }^{30}$ Third, Gli is an effector in the hedgehog signaling pathway in theca cells, which synthesize androstenedione (A4), the precursor of estradiol (E2), which is produced in granulosa cells. We hypothesized that the activation of Gli would correlate with theca cell development in a growing follicle and correlate with the increasing concentrations of produced and secreted sex hormones. And lastly, HIF1 is a hypoxia-induced factor, which is expressed in cells under hypoxic conditions. We hypothesized that HIF1 upregulation would correlate with the growing number of cells and increase in follicle size, followed with the antrum formation.

Upregulation of the aforementioned TFs goes hand-in-hand with follicle expansion and synthesis and secretion of sex hormones: A4, E2, and progesterone (P4). It has been established that in an LHfree, serum-free culture system, theca cells produce A4 under the influence of physiological levels of insulin, ${ }^{8,18,38}$ from which granulosa cells synthesize E2, the two essential hormones of follicle development, according to the "two-hormone two-cell types theory". ${ }^{28}$ Granulosa cells cannot synthesize E2 directly from cholesterol, and theca cells cannot synthesize E2 from A4. The levels of E2 and A4 reach measurable levels at later stages of folliculogenesis, around day 6 of the culture or when follicles reach 200 microns in diameter, ${ }^{36,53}$ confirming the presence and functions of both cell types. The $\mathrm{P} 4$ production in granulosa cells is regulated by follistatin and reaches measurable concentrations at the final stages of folliculogenesis, close to ovulation. Thus we anticipated that in healthy developing follicles, the follicular NF- $\kappa \mathrm{B}$ activities to be associated with E2 production in vitro, ${ }^{13}$ Gli activities with $\mathrm{A} 4$ production, and follistatin with $\mathrm{P} 4 .^{36,53}$ 
In addition to the analysis of TF activities and hormone secretion, we investigated the secretion patterns of 27 cytokines from the family of soluble mediators of cell-to-cell communications ${ }^{64}$ and angiogenesis. A directed ingrowth of new blood vessels, or angiogenesis, is a key process during successful folliculogenesis. ${ }^{56}$ The developing follicle secretes angiogenic factors to attract endothelial cells and improve the influx of oxygen, nutrients and hormones. The newly formed capillaries infiltrate the theca layer when the follicle reaches preantral stages and provide the nutrition. ${ }^{42}$ While the described culture system does not support angiogenesis per se, we hypothesized that a healthy growing follicle would secrete a proangiogenic milieu of factors. We employed data-driven modeling approaches to correlate cytokine activity with TF dynamics, phenotypical changes, and steroidogenesis during in vitro follicle development to reveal any possible unique signatures of $10 \times$ follicles. Taken together, our results provide a panoramic picture of the evolving and complex interplay of molecular networks involved in relaying biochemical cues to the nucleus during successful, number-dependent primary follicle development.

\section{MATERIALS AND METHODS}

\section{Lentivirus and Transcription Factor Arrays}

Lentivirus was produced by co-transfecting HEK$293 \mathrm{~T}$ cells with previously described lentiviral packaging vectors (pMDL-GagPol, pRSV-Rev, PIVS-VSV$\mathrm{G})^{17}$ and lentiviral vectors using JetPrime (Polyplus, Illkirch, France). After $48 \mathrm{~h}$, supernatants were collected and cell debris was spun down and removed. Viruses were concentrated using PEG-it (System Biosciences, Palo Alto, CA) and re-suspended in phosphate buffered saline (PBS).

TF reporters consist of a specific TF response element (TRE) cloned upstream of a minimal thymidine kinase promoter (TA) driving the gene for firefly luciferase (FLuc) or NanoLuciferase (NLuc). TF reporter specificity and sensitivity studies are referenced on the TRANSFAC database. Each lentiviral reporter consists of three or four repeats of a TF-specific binding element driving expression of luciferase.

\section{Follicle Enzymatic Isolation, Transduction and Encapsulation}

To harvest large numbers of primary follicles and to facilitate the subsequent transduction by lentiviruses, we used enzymatic digestion of the ovaries to isolate individual follicles. For all experiments, ovaries were retrieved from female $\mathrm{F} 1$ mice $(\mathrm{C} 57 \mathrm{~B} 6 \times \mathrm{CBA} / \mathrm{J})$ at the age of 10-12 days. After separating from surrounding connective tissues, ovaries were briefly washed with fresh, warm Leibovitz's L-15 medium (L15) (Thermo Fisher) and transferred to a $30 \mathrm{~mm}$ dish containing pre-equilibrated alpha modification of minimum essential medium ( $\alpha$ MEM) (Thermo Fisher) and $0.5 \%(\mathrm{v} / \mathrm{v})$ Pen/Strep (Thermo Fisher). After all the ovaries were transferred, $10 \%(\mathrm{v} / \mathrm{v})$ Liberase DH at 13 Wünsch units/mL (Sigma-Aldrich) was added and gently mixed in the dish. The ovaries were incubated undisturbed for a total of 35-45 min, followed by 5 min of pipetting to break down the enzyme-digested tissues to individual follicles. The enzymatic digestion was then arrested by adding $10 \%(\mathrm{v} / \mathrm{v})$ fetal bovine serum (FBS) (Thermo Fisher).

Immediately after the enzymatic isolation, the digest was treated as a follicle suspension and equally divided into cohorts of follicles to be infected with one of the four transcription factors. To transduce follicles, each cohort of follicles was incubated with approximately 10 multiplicity of infection (MOI) of each TF activityreporting lentivirus for $4 \mathrm{~h}$ in maintenance medium $(\mathrm{MM})$ prior to encapsulation in $0.3 \%(\mathrm{w} / \mathrm{v})$ alginate (Ingredients Solution, Inc., Waldo, ME, USA).

Follicle encapsulation was performed as previously established. Briefly, intact primary follicles at a diameter range of 90-110 $\mu \mathrm{m}$ were selected from the digest and encapsulated in $10 \mu \mathrm{L}$ alginate beads in either five $(5 x)$ or ten $(10 x)$ follicles per bead. Follicle-containing alginate beads were pipetted in $50 \mathrm{mM} \mathrm{CaCl}$ and $140 \mathrm{mM} \mathrm{NaCl}$ and allowed to crosslink for $2 \mathrm{~min}$. The alginate beads were carefully transferred to pre-equilibrated MM to recover for up to $2 \mathrm{~h}$. Alginate beads were individually cultured in 96-well plates, with each well containing $150 \mu \mathrm{L}$ growth media (GM). For each experiment, 4-6 gels per TF were used for either $5 \times$ or $10 \times$ condition. The presented results were pooled from 5 independent cultures. Black walled 96-well plates with optical bottoms were used for bioluminescence measurements to minimize background and cross talk between wells.

NLuc reporters were used to measure luciferase production and correlate transduction efficiency and cell proliferation with production of luciferase. Two days after follicle encapsulation and culture, luciferase production was measured by lysing the follicles. The encapsulated follicles were incubated in culture media with $10 \mathrm{mIU} / \mathrm{mL}$ alginate lyase (Sigma Aldrich) for $2 \mathrm{~min}$ in the incubator. Follicles were removed from the degraded alginate and transferred to Nano-Glo ${ }^{\circledR}$ LCS dilution buffer (Promega) with Reporter Lysis Buffer (Promega). After pipetting to ensure a thorough breakdown of the follicular structures, Nano-Glo ${ }^{\circledR}$ Live Cell substrate was added to the lysate and lumi- 
nescence was immediately measured using GloMax ${ }^{\circledR}$ 20/20 Single Tube Luminometer (Promega), followed by measuring the amount of dsDNA using NanoDrop $^{\text {TM }}$ Spectrophotometer (Thermo Fisher). To monitor the stability of luciferase expression from the normalization vector, we quantified the amount of total DNA and the intensity of bioluminescence by lysing $10 \times$ follicles every 2 days. To avoid potential fluctuations caused by media change and external perturbations, alginate gels were sacrificed approximately $48 \mathrm{~h}$ apart before changing the media. Triplicates of $10 \times$ follicles were used for each time point. Normalized relative luminescence unit (RLU) values were calculated accordingly for each replicate. For transduction efficiency calculation, Day 2 follicles were released from alginate gels and pipetted through SCIENCEWARE $^{\circledR}$ Flowmi $^{\text {TM }}$ Cell Strainers to obtain cell suspension without breaking down cellular structures. Total cell number and luciferase-expressing cells were counted in three separate experiments to estimate the transduction efficiency.

\section{Bioluminescence Quantitative Measurements and Microscopy}

Two days after follicle encapsulation and culture, live-follicle transcription factor activity measurement from 4 different $\mathrm{TF}$ reporters ( $\mathrm{p} 53, \mathrm{NF}-\kappa \mathrm{B}$, Gli, and HIF1) and control (TA) was recorded using a PerkinElmer's IVIS ${ }^{\mathrm{TM}}$ Spectrum imaging system at the Center of Molecular Imaging (CMI) of the University of Michigan. Xenolight luciferin substrate (Perkin Elmer) at $630 \mu \mathrm{M}$ was added to each well $10 \mathrm{~min}$ prior to imaging. Bioluminescence measurements using the IVIS $^{\text {TM }}$ Spectrum (BLI-IVIS) were performed every $24 \mathrm{~h}$ following the first two days of culture. Every 2 days after imaging, half of the media $(75 \mu \mathrm{L})$ was replaced with fresh, pre-equilibrated GM containing luciferin.

For single-cell bioluminescence microscopy (BLIMS), NLuc reporters were used and individual $10 \times$ gels were imaged using an LV200 Bioluminescence Imaging System (Olympus). Gels were imaged in a $30 \mathrm{~mm}$ dish with optical bottom. Sufficient GM containing NanoGlo ${ }^{\circledR}$ Live Cell Substrate was transferred from the original 96-well plate to cover the gel and PBS was added to the surrounding to prevent evaporation. All single-cell BLI-MS images were acquired with a 5minute exposure time and a $200 \mathrm{EM}$ gain. Brightfield and luminescence images were merged using ImageJ. ${ }^{52}$ Individual gels were transferred back to the original 96-well plates for continuous cultures. BLI-MS imaging was performed every $48 \mathrm{~h}$. Immediately after imaging, half of the media $(75 \mu \mathrm{L})$ was collected and replaced with fresh, pre-equilibrated GM.

\section{Hormone and Cytokine Measurements and Analyses}

Follicle-secreted sex hormones: A4, E2, and P4 were analyzed using the collected spent culture media at different time points starting at day 4 of the culture. For each replicate, spent media was pooled from two wells of follicles from the same condition. For hormone assays, four such replicates per condition were independently analyzed by the University of Virginia Center for Research in Reproduction Ligand Assay and Analysis Core. The same set of media samples were used for cytokine measurements. The measurements were performed with the mouse angiogenesis/growth factor kit (MAGPMAG-24 K-27, Milliplex, EMD Millipore Corporation) on a Luminex $200^{\mathrm{TM}}$ system (Millipore Sigma), following manufacturer's instructions with $4 \times$ dilution.

\section{Statistical Analyses}

Statistical analyses for the luciferase production, hormone data, and univariate analyses of cytokines were performed in GraphPad Prism 7. For luciferase production, linear regression was performed. For hormone and univariate cytokine data, two-way ANOVA with repeated measures was performed, followed by Sidak's multiple comparisons tests. Values of $p<0.05$ were considered significant. TF activity data was background subtracted, normalized to the median TA control value and log transformed as previously described. ${ }^{16}$ Differences in means between time points and conditions were evaluated by fitting an empirical hierarchical Bayesian linear model using the limma $\mathrm{R}$ package. ${ }^{58}$ False discovery rate correction was used on the calculated $p$-values. ${ }^{9}$ A $p$ value of $<0.05$ was considered to be statistically significant for the TF activity measurements.

In addition to ANOVA analyses on cytokines, we subsequently employed data-driven modeling approaches. The principal component analysis (PCA) of cytokine data from $5 \times$ and $10 \times$ follicles operates by choosing linear combinations of cytokines (principal components: PCs) that account for most the variation in the overall data set. Each sample can then be plotted on principal components accounting for the most variance (scores plot), with separation in this space indicating distinct signatures that differentiate samples. We also visualized distinguishing signatures by performing unsupervised hierarchical clustering of variable importance projection (VIP) selected cytokine factors. To gain insights to cytokine signatures, we 
used supervised partial least square discriminant analyses (PLSDA) models ${ }^{5,6,37,48}$ and associated VIP scores $^{12}$ to eliminate cytokines that did not contribute to differentiation of the conditions. In the models shown in Figs. 4, 5, and 6, each cytokine at each time point is treated as a unique factor, as opposed to the previous pooling of time points, in order to investigate variation in cytokine relationships across time. Separate correlation networks were created based on only VIP-selected cytokine factors from the PLSDA model in Fig. 4 that were also above the detection limit for all samples in the respective condition. These correlation networks use Pearson correlation coefficients computed for each pair of factors to create a map of significant pairwise relationships.

\section{RESULTS}

\section{Follicles Cultured in Groups of 10 Demonstrated Improved Survival, Growth and Steroidogenesis Compared to Follicles Cultured in Groups of 5}

Primary follicles with diameters ranging between 85 and $100 \mu \mathrm{m}$ and cultured in groups of 10 reached preovulatory sizes (Figs. 1a to 1c), similar to what has been previously reported. ${ }^{26}$ After 12 days of in vitro culture in alginate, $70.0 \%$ of the follicles survived in the $10 \times$ group, compared to a $42.9 \%$ survival rate in $5 \times$ groups (Fig. 1b). Follicles in $10 \times$ groups grew to a larger size, reaching $320 \mu \mathrm{m}$ in diameter on the last day of culture (Figs. 1a and 1c), and a greater number of follicles developed an antrum (Fig. 1a) compared to those cultured in groups of 5. The differences in average follicle diameters became significant as the culture progressed to Day $10(p=0.0017)$ and Day 12 $(p<0.0001)$ (Fig. 1c). Transduction of primary follicles with transcription factor reporters (TFrs) did not affect their development in vitro.

The concentrations of follicle-secreted sex hormones, A4, E2, and P4, increased as the eIVFG progressed and follicles increased in sizes (Figs. 1d to 1f). The estradiol production increased exponentially after follicles reached antral stages. ${ }^{36,53}$ Thus, no significant difference in $\mathrm{E} 2$ levels between $5 \times$ and $10 \times$ groups appeared during the first 8 days of culture. However, the differences between the groups became significant on Day 10 $(p=0.0002)$ and Day $12(p<0.0001)$ of culture, reaching $1.24 \pm 0.16 \mathrm{ng} / \mathrm{mL}$ (D10) and $2.8 \pm 0.31 \mathrm{ng} /$ $\mathrm{mL}$ (D12) respectively. The increasing levels of folliclesecreted E2 correlated with a more successful functional development of follicles cultured in groups of ten, and were consistent with their larger sizes on Day 10 and Day 12. The levels of P4 followed a similar trend with no significant difference between $5 \times$ and $10 \times$ follicles during the first 10 days of culture. On day 12 of eIVFG,
P4 reached $26.35 \pm 6.26 \mathrm{ng} / \mathrm{mL}$, which was significantly higher $(p<0.0001)$ than that in the $5 \times$ follicles $(5.40 \pm 1.60 \mathrm{ng} / \mathrm{mL})$. The differences in A4 levels between $5 \times$ and $10 \times$ follicles remained statistically insignificant at all time points (Fig. 1d).

\section{Dynamic Transcription Factor Activities During Primary Ovarian Follicle Development In Vitro Presented Different Signatures in $10 \times$ and $5 \times$}

Binding of TFs at the TRE results in luciferase production and proportional increase in luminescence, thus providing a quantitative measure of the dynamic activities of TFs in growing follicles (Fig. 2a and b). ${ }^{16}$ BLI provided a rapid, non-invasive, and sensitive method to quantify luciferase levels, and was recorded repeatedly from each sample to monitor dynamic activity. BLI-MS was employed to record bioluminescence at a cellular-level from individual follicles. After 2 days of culture, the luciferase expression in the transduced follicles was localized to the granulosa cells (Fig. 2c). We determined the transduction efficiency of the granulosa cells in primary follicles by counting the number of luciferase-expressing cells, which reached $16.3 \%$ of total cells isolated from the treated follicles (Supplementary Fig. 1A). As anticipated, the amount of luciferase produced by transduced follicles was constant when normalized to the amount of total DNA (Supplementary Fig. 1B), which indicated that transduced and non-transduced granulosa cells were proliferating at a comparable rate.

BLI-IVIS was employed to quantify the bioluminescence at population-level, collecting the signal from wells with multiple follicles. NF- $\kappa \mathrm{B}$, HIF1, Gli and p53 are key TFs that participate in cellular processes of folliculogenesis, such as mechanotransduction, response to hypoxia, angiogenesis, thecal cell differentiation, steroidogenesis, and cell turnover. ${ }^{50,70}$ The activity of NF- $\kappa$ B peaked in $10 \times$ follicles on Day 5 of culture, in contrast to a later peak on Day 8 in $5 x$ follicles (Fig. 2d). The levels of HIF1 (Fig. 2e) in $10 \times$ follicles peaked on Day 6. This peak correlated with antrum formation and the phenotypic observations of follicle expansion, possibly as the result of mechanical stimuli and the hypoxic stress in follicles due to the cell proliferation (Figs. $1 \mathrm{~b}$ and $1 \mathrm{c}$ ). In the $5 \times$ condition, the levels of NF- $\kappa$ B and HIF1 peaked at the same time on Day 8 . Follicles in the $5 \times$ condition had a significantly lower rate of antrum formation and survival. The correlation between NF- $\kappa \mathrm{B}$ and HIF1 peaks and expansion of follicles in $10 \times$ condition supports the hypothesis that hypoxia caused by cell proliferation in follicles leads to the differential antrum formation rates between $5 \times$ and $10 \times$ follicles, and eventually to the differences in survival and terminal sizes. 
D2

(a)

10X

5X
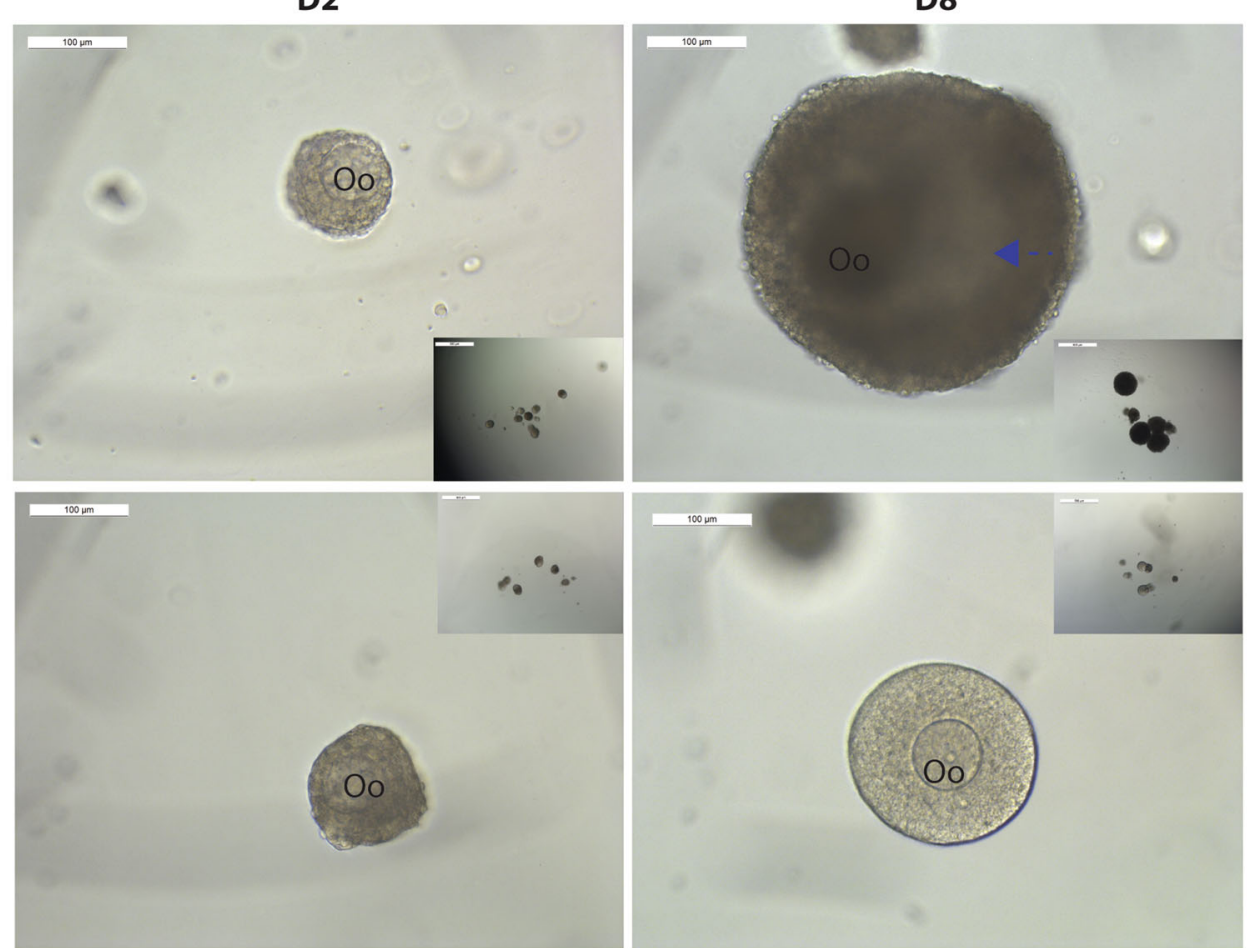

(b)

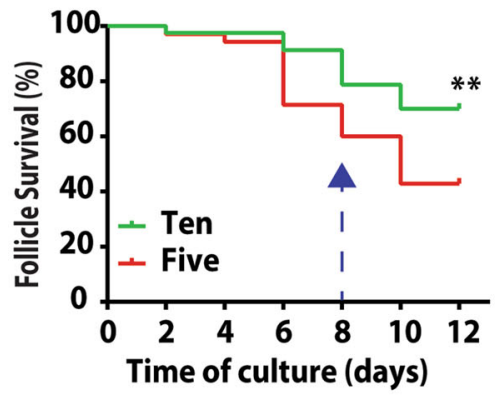

(c)

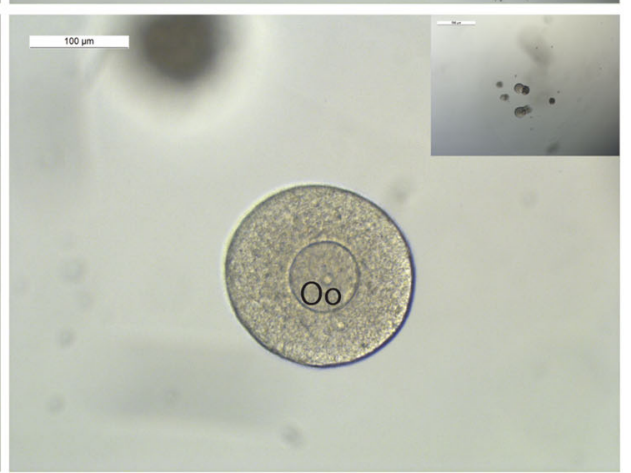

(d)

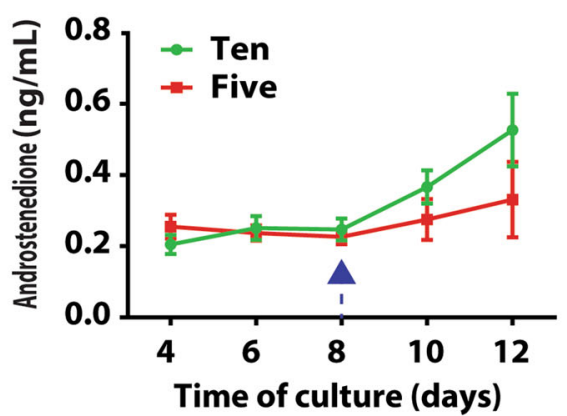

(e)

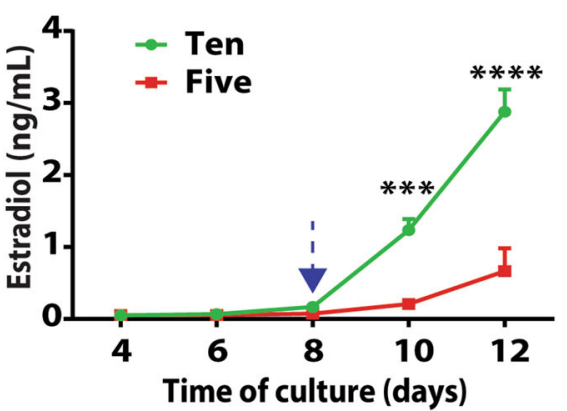

(f)

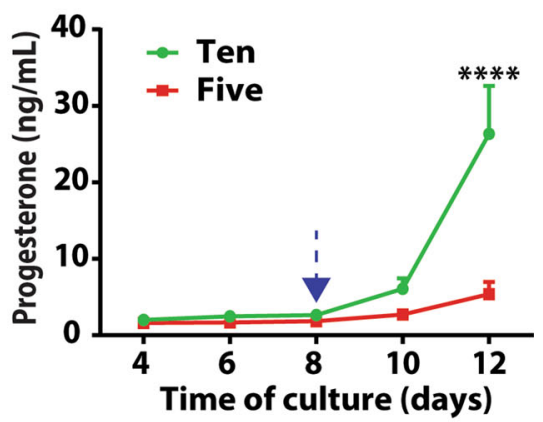

FIGURE 1. The number dependent pattern of primary follicle development in vitro. (a) Representative images of $5 \times$ and $10 \times$ follicles encapsulated in alginate. Scale bar $=100 \mu \mathrm{m}$. Oo: oocyte; blue arrow: antrum; (b) Follicle survival in $5 \times$ and $10 \times$. Sample size: $n=35(5 \times)$ and $80(10 \times)$; (c) Follicle growth in $5 \times$ and $10 \times$. Sample size: $n=15(5 \times)$ and $56(10 \times)$. Data presented as mean \pm SEM; Profiles of androstenedione (A4, d), estradiol (E2, e), and progesterone (P4, $f$ ) in $5 \times$ and $10 \times$ follicles during elVFG. Sample size: $n=4$ per time point per condition. Data presented as mean \pm SEM, ${ }^{\star} p<0.05,{ }^{\star \star} p<0.01,{ }^{\star \star \star} p<0.001,{ }^{\star \star \star \star} p<0.0001$. 
The TF Gli is a member of the hedgehog pathway and induces theca cell differentiation at the later stage of folliculogenesis. ${ }^{79}$ The Gli activity, however, did not peak at any of the time points and had no significant changes between the two conditions (Fig. 2f), which was reflected in the insignificant differences in A4 levels.

Lastly, p53 activity fluctuated during cultures in both $5 \times$ and $10 \times$ conditions (Fig. 2 g), but did not differ significantly between the groups or time points. As a cell cycle regulator, in normal dividing cells, p53 transiently increases in temporal association with checkpoints of cell cycles. ${ }^{31}$ Yet, at the population level of multiple cells in $5 \times$ and $10 \times$ follicles, the fluctuations in the level of p53 had no difference between the conditions.

\section{Multivariate Analysis Identified Signature Cytokine Profiles in $10 \times$ Follicles}

We measured the concentrations of 27 cytokines, growth factors, and chemokines (Supplementary Table 1 ) in the media of $5 \times$ and $10 \times$ eIVFG conditions, using a Lumine ${ }^{\circledR}$ bead-based assay to identify differences in the secretion patterns of extracellular paracrine signals in the two conditions.

The unsupervised principal component analysis (PCA) of cytokine data from $5 \times$ and $10 \times$ conditions revealed distinct signatures and separation of samples by conditions and days in culture (Fig. 3). The first two principal components accounted for $34.1 \%$ of variation in the data. Interestingly, signatures in later stages of folliculogenesis (Days 10-12) in $10 \times$ condition diverged from the early stages (Days 4-6) and from $5 \times$ cultures at all the time points, supporting the differences in TF activities, steroidogenesis, survival and phenotypes, while indicating that further use of supervised data-driven approaches may give insight into the timing of cytokine interactions that may drive these differences.

\section{The Concentrations of Cytokines Secreted in $10 \times$ Follicles Conditions Differed From $5 \times$ Follicles}

The univariate analyses of cytokine levels identified significant differences between $5 \times$ and $10 \times$ conditions (Figs. 4a to $4 \mathrm{~h}$ and Supplementary Fig. 2) for 8 cytokines, among which $75 \%$ were angiogenesis-related with the remaining $25 \%$ being macrophage-related.

The levels of ovarian angiogenic factors, including $\mathrm{KC} / \mathrm{CXCL} 1$ (keratinocyte-derived chemokine) (Fig. 4a), Angiopoietin-2 (ANGPT-2, Fig. 4b), vascular endothelial growth factor A (VEGF-A, Fig. 4c), and "cell mobilizers" such as granulocyte colonystimulating factor (G-CSF, Fig. 4d) and placental growth factor (PLGF-2, Fig. 4e) were significantly
4FIGURE 2. Lentiviral transduction of enzymatically isolated ovarian follicles and the dynamic activities of transcription factors. (a) Construct of the luciferase reporter. TRE: transcription factor respond element; TA: tyrosine kinase promoter; (1) fusion of the lentivirus to granulosa cells; (2) reverse transcription; (3) integration to host cell genome; (4) specific transcription factors translocating to the nucleus; (5) and (6) start of gene transcription; (7) translation of the luciferase protein; (8) detection of luminescence. (b) Illustration of lentiviral transduction of enzymatically isolated ovarian follicles; (c) representative bioluminescence images of transduced follicles to confirm luciferase expression. Scale bar $=100 \mu \mathrm{m} ;(\mathrm{d}-\mathrm{g})$ dynamic activities of selected TFs (NF- $\kappa \mathrm{B}=\mathrm{d}, \mathrm{HIF} 1=\mathrm{e}, \mathrm{Gli}=\mathrm{f}$, and $\mathrm{p} 53=\mathrm{g}$ ) during folliculogenesis.

higher in $10 \times$ follicles compared to $5 \times$ follicles, indicating that proliferation-induced angiogenesis is probably controlled by the interaction of paracrine factors produced by granulosa cells. Our findings demonstrated that during gonadotropin-dependent, or later, follicular growth, the increased levels of angiogenic factors coincide with the formation of antrum. This evidence supports the hypothesis that although angiogenesis takes place in thecal layer, granulosa cells exert an important role in this process by producing key angiogenic factors. ${ }^{63,80}$

Levels of an important group of cytokines, such as follistatin, IL-6, and monocyte chemotactic protein-1 (MCP-1), related to oocyte maturation were significantly higher $(p<0.0001)$ in $10 \times$ follicles compared to $5 \times$ follicles (Figs. 4f to $4 \mathrm{~h}$ ). Follistatin prevents uncontrolled proliferation of granulosa cells in preantral follicles and is associated with further differentiation of granulosa cells as follicles mature and prepare for ovulation and post-ovulatory progesterone production (Figs. 1c, 1e, 1f and 4f). ${ }^{20}$ Similarly, the pro-survival macrophage-related factor, IL-6 that is associated with healthy growing follicles in the prepubertal ovary ${ }^{22,65}$ and serves as a potent autocrine regulator of ovarian cumulus cell function, ${ }^{39}$ was significantly higher in $10 \times$ follicles and correlated with greater maturation competency of oocytes. ${ }^{33}$ The elevated levels of follistatin, IL-6, and MCP-1in the $10 \times$ condition correlate with the increased $\mathrm{P} 4$ production, which further supports our findings that follicles in the $10 \times$ condition have greater maturation competency.

The Differential Cytokine Profiles of $5 \times$ and $10 \times$ Follicles Reveal Synergism in $10 \times$ Follicles Not Present in $5 \times$ Follicles

Using a PLSDA model based on VIP-selected cytokines, we investigated whether the improved outcomes of folliculogenesis in $10 \times$ vs. $5 \times$ was a result of synergism or a simple additive effect. The distinct signature of the cytokine profiles in $10 \times$ follicles (Fig. 5a) was significantly different and separated from the cy- 
(a)

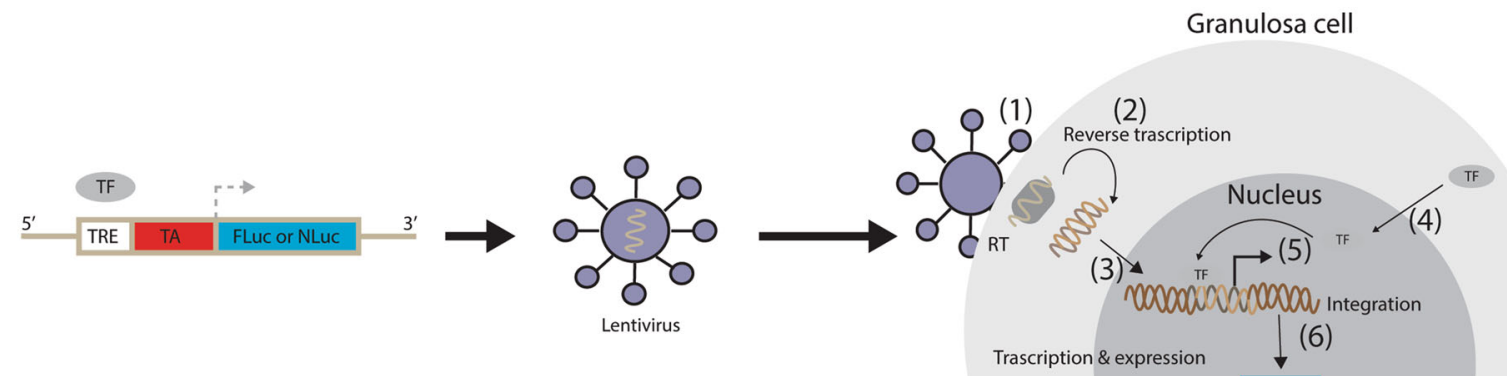

(b)
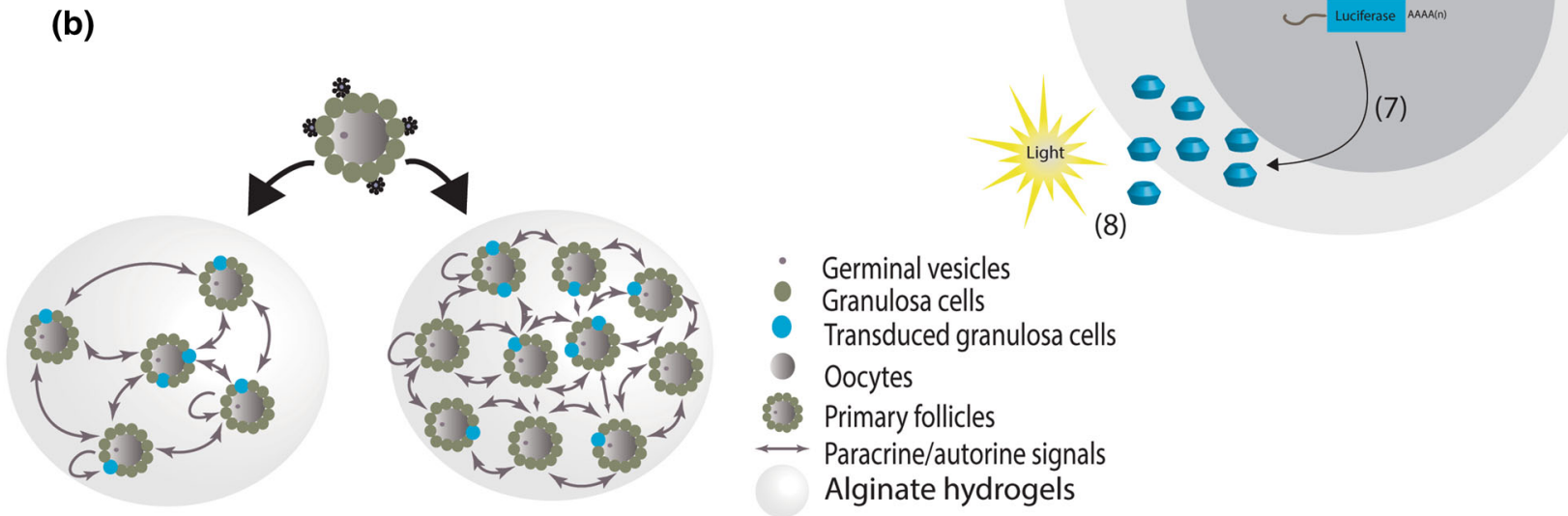

(8)

- Germinal vesicles

- Granulosa cells

- Transduced granulosa cells

Oocytes

:. Primary follicles

$\rightarrow$ Paracrine/autorine signals Alginate hydrogels
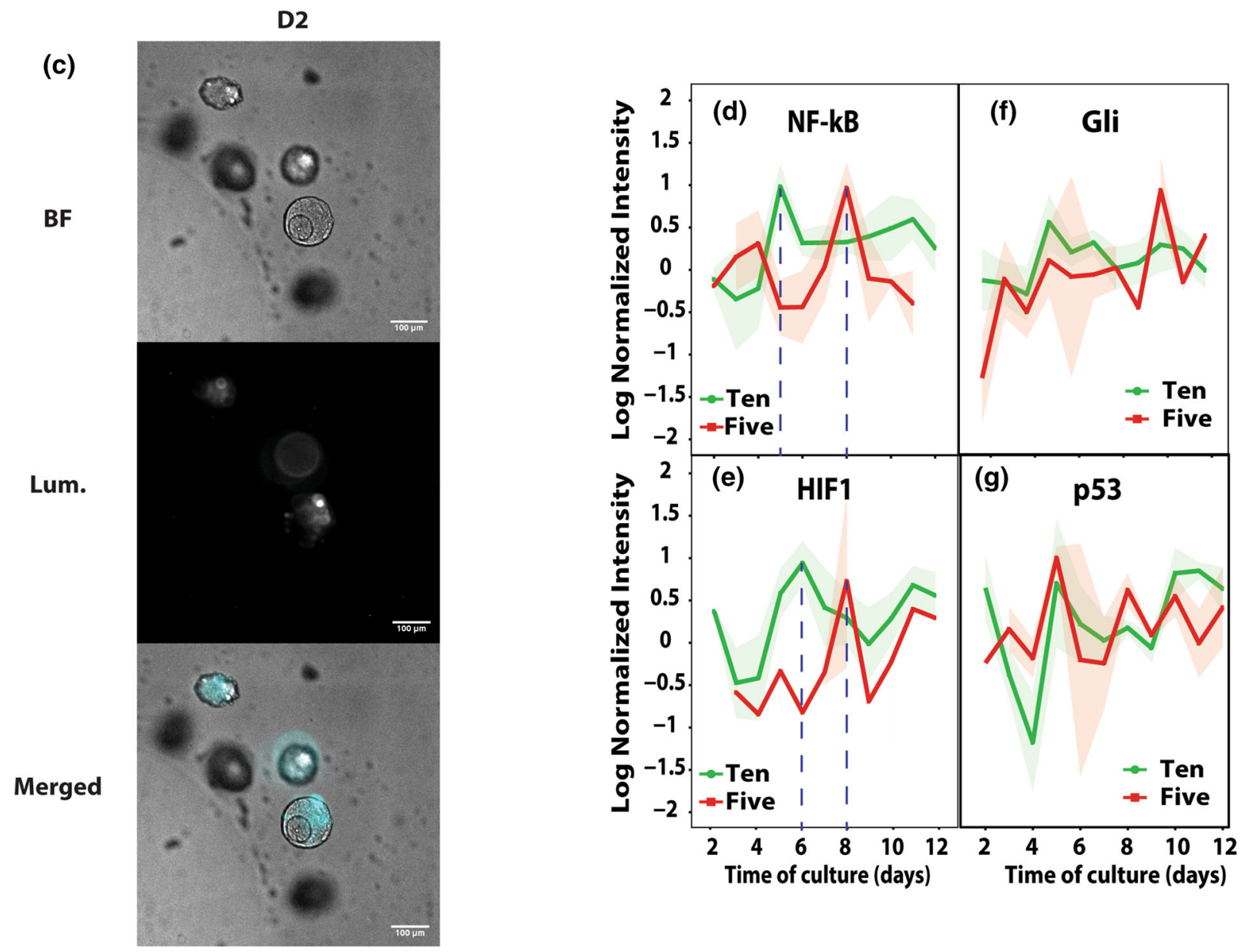
tokine profile in $5 \times$ and $5 \times$ doubled at all time points. This model was able to classify samples by conditions with a calibration error of $0.00 \%$ and a cross-validation error of $2.08 \%$. We further visualized distinguishing signatures by performing unsupervised hierarchical clustering on the VIP-selected cytokine factors (Fig. 5b). Samples generally clustered by culture condition $(5 \times, 10 \times$, or $5 \times$ doubled). Interestingly, this analysis indicated that doubling the concentrations measured in $5 \times$, e.g., $5 \times$ doubled conditions, were distinct from all the $10 \times$ measurements, suggesting cytokine upregulation in $10 \times$ was beyond what was expected by simple additive effect. The differentiating cytokines were grouped into two major clusters, one with upregulation primarily in $10 \times$ samples, and the other with upregulation primarily in $5 \times$ doubled samples. The predominant patterns being upregulation

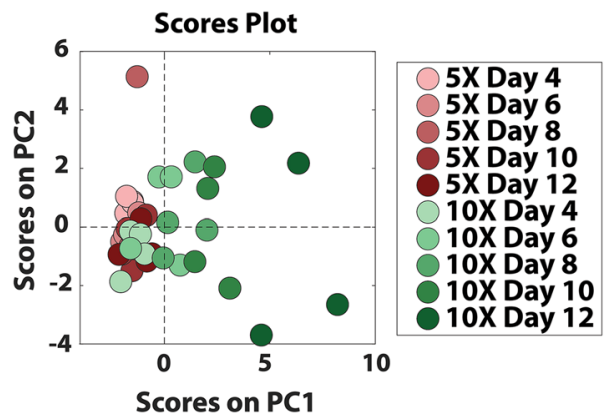

FIGURE 3. Unsupervised principle analyses of cytokines in $5 \times$ and $10 \times$ conditions. Sample size: $n=4$ per time point per condition. exclusively in $10 \times$ or $5 x$ doubled confirm that the number-dependent pattern of growth and survival is due to synergistic effects of higher follicle number.

In order to gain insight into the synergistic phenomenon, we made a separate VIP-selected PLSDA model with only the $10 \times$ and $5 \times$ doubled conditions (Fig. 6). Thirty-nine VIP-selected factors gave perfect separation, with calibration and cross validation errors of $0.00 \%$ using a single latent variable (Fig. 6a). Twenty-seven out of the thirty-nine VIP-selected factors were positively loaded on LV1, indicating positive association with $10 \times$ follicles, relative to the remaining negatively loaded factors which were associated with the theoretical $5 \times$ doubled condition (Fig. 6b). The first latent variable was able to account for $51.21 \%$ of the variance between the two conditions (Fig. 6a). The subsequent loadings plot (Fig. 6b) displayed cytokines from which significance arose, confirming what we identified as significant in univariate analysis (Figs. 4a to $4 \mathrm{~h}$ ), as well as incorporating co-varying cytokines that were dismissed by univariate analysis (Supplementary Fig. 2).

Cytokine Profiles of $5 \times$ and $10 \times$ Follicles Suggest That Early Interactions Between Cytokines Are Critical for Successful Development of Primary Follicles In Vitro

With the goal of identifying trends in bivariate relationships between factors that could give insight into mechanism, we created separate correlation net-
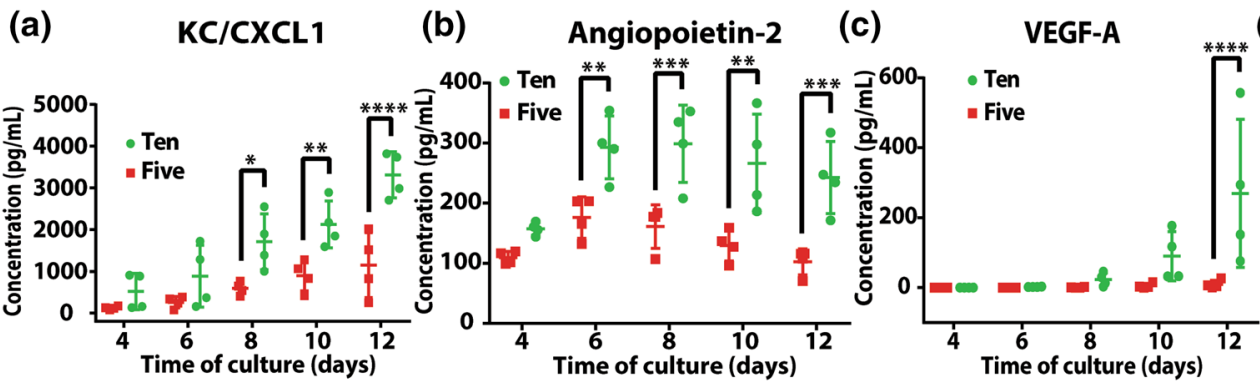

(d)

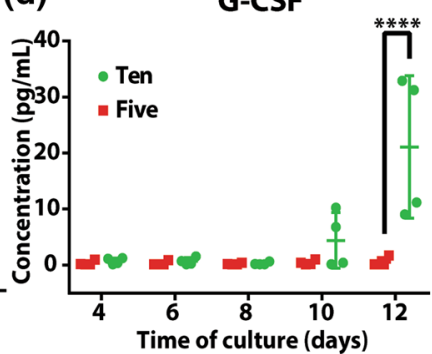

(e)

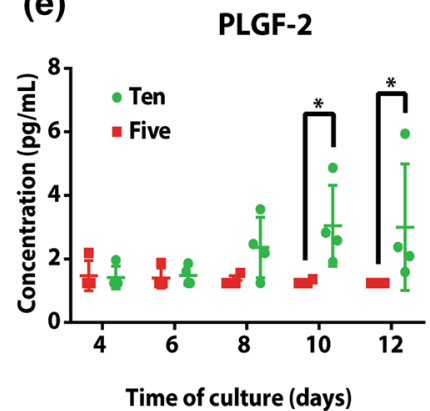

(f)

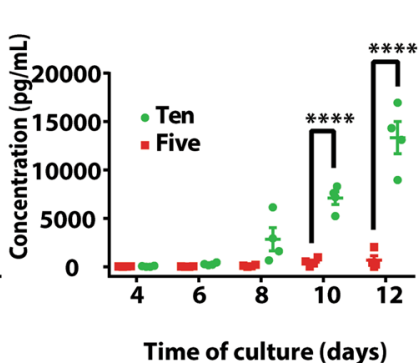

(g)

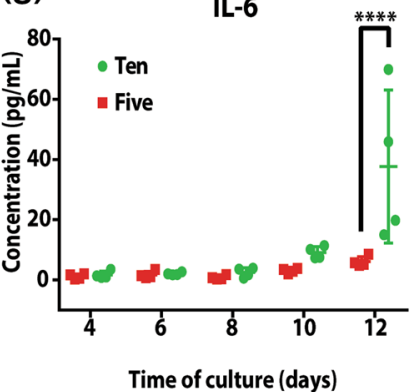

(h)

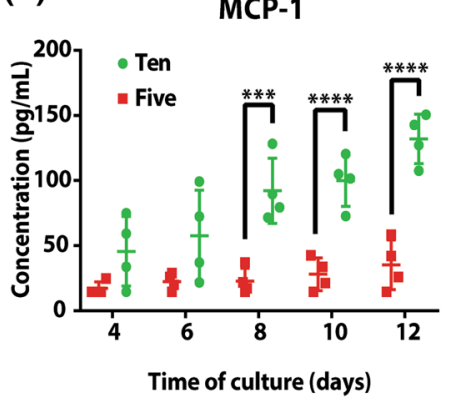

FIGURE 4. Univariate analyses of cytokines of $5 \times$ and $10 \times$ follicles in vitro. Data presented as mean \pm SD, ${ }^{*} p<0.05$, ${ }^{\star *} p<0.01$, ${ }^{\star * *} p<0.001,{ }^{* * \star *} p<0.0001$. 


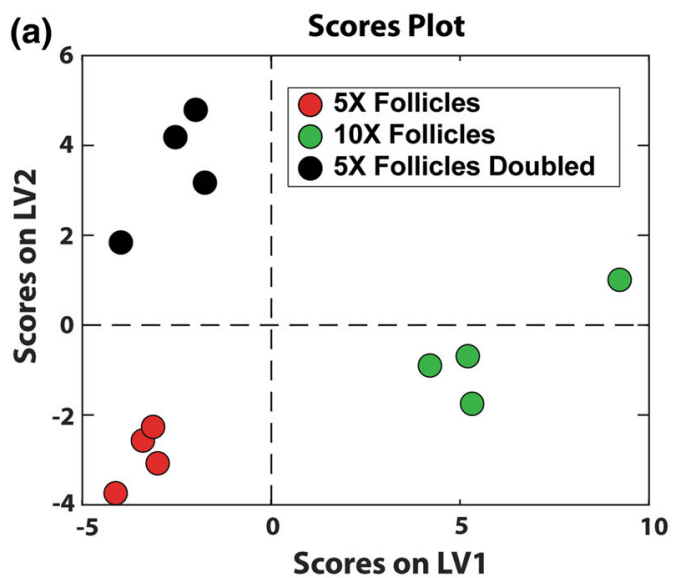

(b)

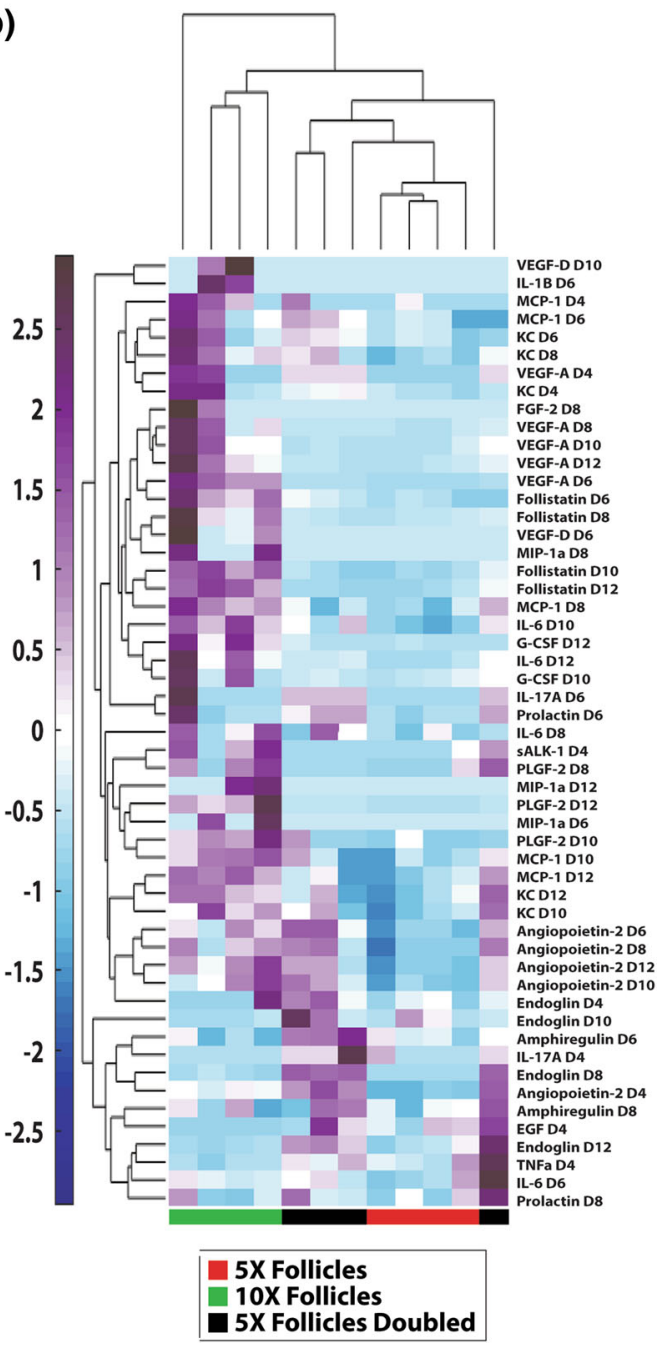

FIGURE 5. Synergism between follicles in the $10 \times$ condition. Sample size: $\mathrm{n}=\mathbf{4}$ per time point per condition. (a) Partial least square discriminant analyses on cytokines identified signatures of $10 \times$ follicles. (b) Clustered heat maps of cytokines revealed different patterns of cytokines in $10 \times$ follicles, $5 \times$ follicles and $5 \times$ follicles doubled. The normalized cytokine abundance was shown in the color bar to the left of the hierarchical cluster (purple denotes relative upregulation and blue denotes relative downregulation in relation to the mean). works for both $5 \times$ and $10 \times$ follicle systems using the VIP-selected cytokine measurements chosen by the $5 x$ vs. $10 \times$ vs. $5 \times$ doubled PLSDA model that were above the detection limit for all samples (Fig. 7). These correlation networks use Pearson correlation coefficients computed for each pair of factors to create a map of significant pairwise relationships. Intriguingly, our networks indicated a great density of correlations early in the $10 \times$ follicles (Day 6 and 8, Fig. 7a), which were not present in the $5 \times$ follicles (Fig. $7 b$ ). The $5 \times$ condition had a greater density of correlations much later, around days 10 and 12 of the culture, which was consistent with the overall delayed development and lack of cytokines synergism. The timing of a greater density of correlation in the $10 \times$ condition overlaps with antrum formation, and suggested that early synergistic effects improve overall survival of the follicles.

\section{DISCUSSION}

Paracrine interactions between the extracellular environment and cells are crucial for successful and coordinated development, especially for complex multicellular units such as ovarian follicles. Using TRACER and cytokine analysis, we identified critical interactions between cytokines and TFs which appeared early in the culture and correlated with phenotypical and functional differences between $5 \times$ and $10 \times$ follicles that appeared later. Furthermore, the multivariate analyses revealed differential profiles of cytokine secretion between the two conditions, which cannot be explained by simple additive effect. By comparing to the expected $10 \times$ concentrations of cytokines established by doubling $5 \times$ concentration, we discovered, for the first time, a distinct signature of the actual $10 \times$ measurements, suggesting that the successful development of $10 \times$ follicles was the result of synergism between secreted cytokines, chemokines, and hormones. The discovered synergism confirmed the need of paracrine support for individual primary follicles as evidenced in various in vitro cultures. $^{26,39,61,65}$ Lastly, our non-invasive approach allowed us to monitor cytokine/chemokine and TF levels in the same follicles over time providing realtime, continuous measurements coupled to the dynamic changes of the growing follicles.

In order for extracellular stimuli, such as oxygen tension or mechanical stiffness, to be transduced to the intracellular compartment, cytokines, chemokines, and growth factors need to trigger signaling cascades that ultimately activate transcription factors. Our results identified a unique synchrony between the quantity and temporal expression of TFs and cytokines, which correlated with successful number-dependent develop- 

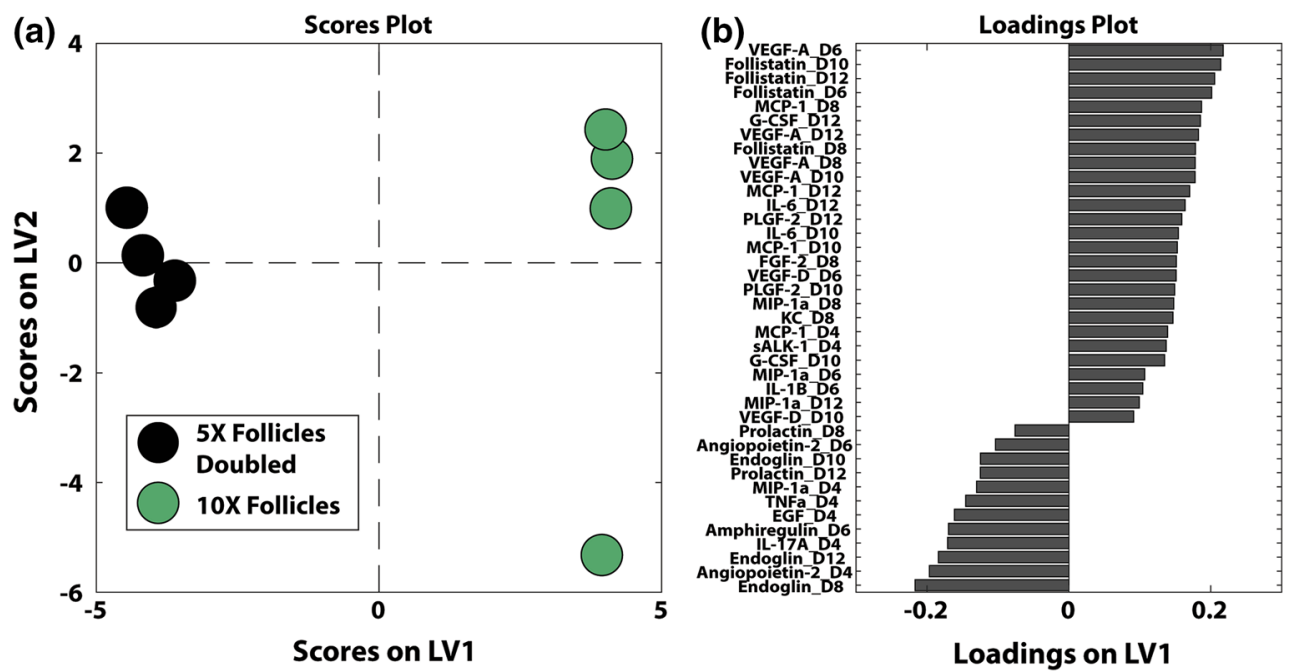

FIGURE 6. Supervised partial least square analyses (a) on cytokine profiles between $10 \times$ follicles and $5 \times$ follicles doubled to identify factors (b, based on latent variables) distinguishing actual $10 \times$ and the expected $(5 \times$ doubled), displaying the synergism in the $10 \times$ conditions.

(a)

10X Correlation Network

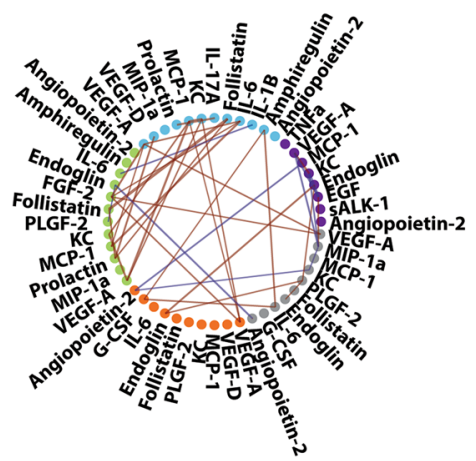

(b)

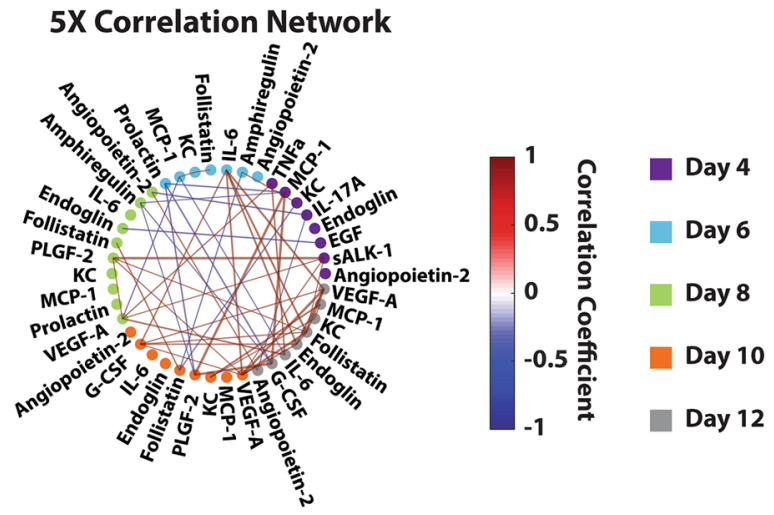

FIGURE 7. Correlation maps of $10 \times(a)$ and $5 \times(b)$ conditions, suggesting earlier interaction of factors in $10 \times$ follicles, indicative of potential intervention regimes. Correlation networks were created based on only VIP-selected cytokine factors from the PLSDA model in Fig. 4 that were also above the detection limit for all samples in the respective condition. Each node (dot) represents a cytokine factor, and each edge (line connecting nodes) represents the correlation between the two nodes. Nodes are colored by day (day 4 as purple, day 6 as cyan, day 8 as green, day 10 as orange, day 12 as grey). Edge thickness represents the significance of the bivariate correlation, thicker being more significant. Edge color represents the correlation coefficient, indicating the direction of correlation (red denoting positive and blue denoting negative). All correlations with a $p$-value below 0.02 are not shown.

ment of early stage follicles. For example, the mechano- and oxygen-responsive TFs, NF- $\kappa$ B and HIF1, exhibited a unique upregulation signature in $10 \times$ follicles. We observed an increase in NF- $\kappa \mathrm{B}$ levels in $10 \times$ follicles on Day 5 of culture, accompanied by levels of HIF1 in $10 \times$ follicles subsequently significantly increasing ( $p=0.013$, compared to Day 4) 1 day later, indicating the hypoxic stress in the growing follicles as a consequence of cell proliferation and a possible "need" to send signals that promote angiogenesis. NF$\kappa \mathrm{B}$ takes part in the regulation of the granulosa cell fate during ovarian follicular development ${ }^{14,69}$ and is responsive to substrate stiffness. ${ }^{23,29}$ As a result of cytokine-induced granulosa cell proliferation, the transcriptional activities of $\mathrm{NF}-\kappa \mathrm{B}$ significantly increased in $10 \times$ follicles, consistent with observed improved survival and growth of $10 \times$ follicles. Consequently, the proliferating granulosa cells may create higher oxygen tension, leading to hypoxia in the local environment. Specifically for follicles, it has been shown that hypoxic condition is an integral part for the survival and growth of early secondary stage follicles in vitro ${ }^{41}$ and is essential for the initiation of the antrum formation, which is consistent with our results. Additionally, the levels of HIF1 increased before antrum formation and immediately decreased afterwards, 
indicating that follicles experienced hypoxia until antrum formation, which was relieved at the antral stage. Consequently, the $10 \times$ follicles showed improved survival and growth, which was further reinforced with a unique signature of TF activity compared to $5 \times$ follicles.

In vivo, as primary follicles continue to increase their diameter as a result of granulosa cell proliferation, growing follicles form their own individualized vascular supply through activation of a synergistic network $^{24}$ under the influence of oxygen tension. Specifically, VEGF-A appeared early and often in the multivariate $10 \times$ signatures (Fig. 5 b: associated with all the time points and Fig. 6: associated with all the time points after day 4), indicating its synergistic role in the $10 \times$ follicles, and showed significant difference on day 12 in the univariate analysis (Fig. 4c). Interestingly, the significant difference of ANGPT-2 (Fig. 4b) appeared to be additive between $5 \times$ and $10 \times$ follicles as indicated by the expected and actual $10 \times$ follicles (Figs. 5b and 6), possibly allocating a more central role to VEGF-A in this synergistic network. Consistent with TF activities, levels of VEGF-A and angiopoietin-2 (ANGPT-2) were significantly higher in $10 \times$ compared to $5 \times$ follicles. It has been previously established that VEGF-A accumulation in media was positively correlated with follicle growth rate in vitro ${ }^{21}$ and that VEGF-A levels increase after antrum formation. ${ }^{73}$ Similarly, in vitro-grown follicles produce increasing concentrations ANGPT-2 after antrum formation, especially follicles displaying considerable estrogenic activity and producing mature oocytes. ${ }^{21,77}$ Our VEGF-A and ANGPT-2 results (Figs. 4b and 4c) followed the same trend, which led us to hypothesize that in mouse, the symbiotic relationship between elevated concentrations of VEGF-A and ANGPT-2 allows follicles to develop the vascular network and progress to maturation after antrum formation.

The critical role of angiogenesis in follicular function, including steroidogenesis and oocyte maturation, is evident in the activity of other local angiogenic factors that we discovered in this study. For example, the angiogenic factor $\mathrm{KC}$ displays significant differences between $5 \times$ and $10 \times$ conditions. As an inducible transcription factor related to angiogenesis at early time points, ${ }^{59} \mathrm{KC}$ was more distinctly associated with $10 \times$ follicles in early days (Fig. 6). The early association of $\mathrm{KC}$ could synergistically contribute to the elevated levels of the potent mitogen VEGF-A, stimulating the proliferation in the follicular structure and migration of the blood vessel precursor cells. Moreover, the increased pro-angiogenic cues consequently led to significant increases in levels of possibly downstream effectors of ANGPT-2, KC, and VEGFA, or "cell mobilizers", such as G-CSF and PLGF$2 .^{44,57}$ Indeed, G-CSF showed significant differences between $5 \times$ and $10 \times$ follicles in univariate analysis on day 12 (Fig. 4d) and appeared in $10 \times$ signatures for both PLSDA models (Figs. 5 and 6) on days 10 and 12, indicating its synergistic effect during follicle development in the $10 \times$ condition. Similarly, matching the significant differences between $5 \times$ and $10 \times$ follicles on days 10 and 12 in univariate analysis (Fig. 4e), PLGF-2 was associated with $10 \times$ (Figs. 5 and 6) as early as day 8. As a member of the VEGF family, PLGF-2 binds only to VEGF receptor 1 (VEGFR1). Consistent with previous reports on roles of PLGF-2 in early follicle development and angiogenesis, ${ }^{1,34,66}$ our results also indicated its synergistic enhancement of the survival and growth of $10 \times$ follicles.

However, in our 3D alginate culture system, the encapsulated follicles cannot develop new blood vessels and increase the supply of nutrients, as would happen in vivo in response to all the secreted angiogenic factors. The inability of theca cells to exchange signals with migrating endothelial cells and the granulosa cells to experience the increased influx of hormones, oxygen and nutrients presents a certain limitation in the current setup. New generation culture systems $^{43,55,62}$ that incorporate degradable matrices and allow co-encapsulation of endothelial and other cells with the growing follicles will address some of these limitations in the future.

Nevertheless, our results support the other reports in the field regarding the association between angiogenesis and follicle development. ${ }^{60}$ Growing follicles secrete sex hormones, such as E2, A4 and P4, during the later gonadotropin-dependent stages of folliculogenesis. The exponential increase of E2 levels in $10 \times$ follicles implies the presence of functioning theca cells that produce A4, the precursor for E2, and granulosa cells that complete the synthesis, despite of the lack of Gli peaks and the insignificant differences in A4 levels, even though the A4 levels were comparable to previously established. ${ }^{36,53}$ One explanation for the lack of significant differences in Gli activity in the described system is the low number of theca cells present and transduced in isolated primary follicles at the beginning of the experiment, which subsequently results in lower number of theca cells that contribute to the BLIIVIS signal, but not necessarily small number of functioning theca cells overall. Additionally, the insignificant differences in A4 levels between conditions could indicate a limited theca cell de novo formation $^{38}$ as a result of a low, rate-limiting vascularization, ${ }^{60}$ which stems from the lack of endothelial cells in the current eIVFG.

The activity of NF- $\kappa$ B peaked in $10 \times$ follicles on Day 5 of culture, in contrast to a later peak on Day 8 in $5 \times$ follicles (Fig. $2 \mathrm{~d}$ ). NF- $\kappa \mathrm{B}$ is responsive to substrate stiffness ${ }^{23,29}$ and controls the expression of gene 
products that affect important cellular processes, such as cell survival, cell cycle, and apoptosis. ${ }^{30,78}$ Substrate stiffness plays a role during folliculogenesis, because follicles experience compressive forces from the environment as they expand in alginate. ${ }^{72}$ Interestingly, the levels of HIF1 (Fig. 2e) in $10 \times$ follicles significantly increased $(p=0.013)$ compared to Day 4, on Day 6 of culture, after the NF- $\kappa \mathrm{B}$ peak and right around the time of antrum formation, correlating with the phenotypic observations of follicle expansion, leading to mechanical stimuli and the hypoxic stress in follicles as the consequence of cell proliferation (Figs. $1 \mathrm{~b}$ and $1 \mathrm{c}$ ). In contrast to the sequential activity of NF- $\kappa \mathrm{B}$ and HIF1 in $10 \times$ follicles, the levels of NF- $\kappa$ B and HIF 1 peaked at the same time on Day 8 in $5 \times$ follicles. Consequently, follicles in the $5 \times$ condition had significantly lower rates of antrum formation and survival. The correlation between NF- $\kappa \mathrm{B}$ and HIF1 peaks and expansion of follicles in $10 \times$ condition supports the hypothesis that hypoxia caused by cell proliferation in follicles leads to the differential antrum formation rates between $5 \times$ and $10 \times$ follicles, and eventually to the differences in survival and terminal sizes.

Consistent with the rest of univariate analysis, the data-driven multivariate analysis identified additional cytokines that appeared to be synergistic in $10 \times$ follicles, including follistatin, MCP-1, and IL-6 (Figs. 4, 5, and 6). Apart from the significant differences present in their levels between $5 \times$ and $10 \times$ follicles, these synergistic cytokines also shared early association with $10 \times$ follicles (Figs. 5 and 6). Particularly, NF- $\kappa \mathrm{B}$ has been shown to be involved in IL-6 release mediated by MCP-1 in vascular smooth muscle cells, ${ }^{68}$ which may promote the development of vasculature in ovarian follicles. ${ }^{45}$ As a peripheral and local regulator, follistatin was identified to oppose the effects of activin, which promotes the development and maintenance of healthy follicles and prevents premature luteinization. ${ }^{19}$ Our data was consistent with its functions in vivo. Taken together, early association of cytokines and growth factors seems to lead to the significant differences at later time points observed in $10 \times$ follicles, as shared by all factors that appeared to be synergistic. This synergism and proper timing of taking effects could contribute to the significant differences in follicle survival, growth and maturation as observed in $5 \times$ and $10 \times$ follicles.

In addition to show what factors and at what levels they are necessary for successful primary ovarian follicle development in vitro, our results suggested that timing of the interactions between those identified factors was critical for the progression of follicles in vitro. Similar to previously reported spatio-temporal profiles for the developmental stages of early folliculogenesis, ${ }^{10}$ our analysis highlighted the in vitro course of paracrinecontrolled process. The early elevated interactions between factors support the transitional state of primary follicles from paracrine-controlled growth mode to endocrine-controlled one. ${ }^{24}$ Taken together with the fact that individually cultured later stage secondary follicles produce mature oocytes in various eIVFG systems, ${ }^{44,54,55,57,59}$ the intervention of adding exogenous factors to support individual primary follicles may only be necessary before secondary stage.

In conclusion, we investigated the number-dependent manner of mouse primary ovarian follicle development in vitro at the cytokine and transcriptional levels. Our novel systems-level, integrated experimental approach captured the complexity of mechanistic pathways that leads to the phenotypical and functional differences in $5 x$ and $10 \times$ follicles. The discovered novel synergism is a significant step forward towards understanding the underlying mechanisms that dictate the relationship between the extracellular microenvironment and downstream effects on individually cultured primary follicle in vitro. Correlations of TRACER, cytokine, and hormone results with confirmation in phenotype and functionality changes serve as a useful in vitro model to molecularly dissect media components and their effects on folliculogenesis across species. This strategy show great potential in developing new in vitro eIVFGs and the molecular-level understanding that may ultimately enable the development of cell-free, serum-free media for follicles at all developmental stages across species.

\section{ELECTRONIC SUPPLEMENTARY MATERIAL}

The online version of this article (https://doi.org/10. 1007/s12195-018-0545-8) contains supplementary material, which is available to authorized users.

\section{ACKNOWLEDGMENTS}

The authors would like to acknowledge the University of Virginia Center for Research in Reproduction Ligand Assay and Analysis Core (Eunice Kennedy Shriver NICHD/NIH (NCTRI) Grant P50HD28934) for performing the hormone assays. This work was supported by The Graduate Assistance in Areas of National Need (GAANN) Fellowship to CET, R01 CA214384 to LDS and the NSF CAREER Award (\#1552580) to AS.

\section{CONFLICT OF INTEREST}

Hong Zhou, Joseph T. Decker, Melissa M. Lemke, Claire E. Tomaszweski, Lonnie D. Shea, Kelly B. Ar- 
nold, and Ariella Shikanov declare that they have no conflicts of interest.

\section{ETHICAL STANDARDS}

No human studies were carried out by the authors for this article. All animal studies were carried out in accordance with guidelines approved by The Institutional Animal Care and Use Committee (IACUC) at the University of Michigan.

\section{REFERENCES}

${ }^{1}$ Abir, R., et al. Vascular endothelial growth factor A and its two receptors in human preantral follicles from fetuses, girls, and women. Fertil. Steril. 93(7):2337-2347, 2010.

${ }^{2}$ Agarwal, S. K., et al. Leptin antagonizes the insulin-like growth factor-I augmentation of steroidogenesis in granulosa and theca cells of the human ovary. J. Clin. Endocrinol. Metab. 84(3):1072-1076, 1999.

${ }^{3}$ Aguado, B. A., et al. Secretome identification of immune cell factors mediating metastatic cell homing. Sci. Rep. 5:17566, 2015.

${ }^{4}$ Ahsan, S., M. Lacey, and S. A. Whitehead. Interactions between interleukin-1 beta, nitric oxide and prostaglandin E2 in the rat ovary: effects on steroidogenesis. Eur. J. Endocrinol. 137(3):293-300, 1997.

${ }^{5}$ Arnold, K. B., and A. W. Chung. Prospects from systems serology research. Immunology 153(3):279-289, 2017.

${ }^{6}$ Arnold, K. B., et al. CD4+ T cell-dependent and CD4+ T cell-independent cytokine-chemokine network changes in the immune responses of HIV-infected individuals. Sci. Signal 8(399):104, 2015.

${ }^{7}$ Baka, S., and A. Malamitsi-Puchner. Novel follicular fluid factors influencing oocyte developmental potential in IVF: a review. Reprod. Biomed. 12(4):500-506, 2006.

${ }^{8}$ Barbieri, R. L. Insulin stimulates androgen accumulation in incubations of minced porcine theca. Gynecol. Obstet. Investig. 37(4):265-269, 1994.

${ }^{9}$ Benjamini, Y., and Y. Hochberg. Controlling the false discovery rate: a practical and powerful approach to multiple testing. J. R. Stat. Soc. Ser. B 57(1):289-300, 1995.

${ }^{10}$ Bonnet, A., et al. Spatio-temporal gene expression profiling during in vivo early ovarian folliculogenesis: integrated transcriptomic study and molecular signature of early follicular growth. PLoS ONE 10(11):e0141482, 2015.

${ }^{11}$ Bornstein, S. R., H. Rutkowski, and I. Vrezas. Cytokines and steroidogenesis. Mol. Cell. Endocrinol. 215(1):135-141, 2004.

${ }^{12}$ Chong, I.-G., and C.-H. Jun. Performance of some variable selection methods when multicollinearity is present. Chemom. Intell. Lab. Syst. 78(1):103-112, 2005.

${ }^{13}$ Chou, C. H., and M. J. Chen. The Effect of steroid hormones on ovarian follicle development. Vitam. Horm. 107:155-175, 2018

${ }^{14} \mathrm{Chu}$, S., et al. Transrepression of estrogen receptor beta signaling by nuclear factor-kappab in ovarian granulosa cells. Mol. Endocrinol. 18(8):1919-1928, 2004.

${ }^{15}$ Conti, M., et al. Role of the epidermal growth factor network in ovarian follicles. Mol. Endocrinol. 20(4):715-723, 2006.
${ }^{16}$ Decker, J. T., et al. Systems analysis of dynamic transcription factor activity identifies targets for treatment in Olaparib resistant cancer cells. Biotechnol. Bioeng. 114(9):2085-2095, 2017.

${ }^{17}$ Dull, T., et al. A third-generation lentivirus vector with a conditional packaging system. J. Virol. 72(11):8463-8471, 1998.

${ }^{18}$ Erickson, G. F., et al. The effects of insulin and insulin-like growth factors-I and-II on estradiol production by granulosa cells of polycystic ovaries. J. Clin. Endocrinol. Metab. 70(4):894-902, 1990.

${ }^{19}$ Findlay, J. K. Peripheral and local regulators of folliculogenesis. Reprod. Fertil. Dev. 6(2):127-139, 1994.

${ }^{20}$ Findlay, J. K., et al. Recruitment and development of the follicle; the roles of the transforming growth factor- $\beta$ superfamily. Mol. Cell. Endocrinol. 191(1):35-43, 2002.

${ }^{21}$ Fisher, T. E., et al. Vascular endothelial growth factor and angiopoietin production by primate follicles during culture is a function of growth rate, gonadotrophin exposure and oxygen milieu. Hum. Reprod. (Oxford, England) 28(12):3263-3270, 2013.

${ }^{22}$ Foster, R., et al. A differential cytokine expression profile is induced by highly purified human menopausal gonadotropin and recombinant follicle-stimulating hormone in a pre- and postovulatory mouse follicle culture model. Fertil. Steril. 93(5):1464-1476, 2010.

${ }^{23}$ Hahn, C., and M. A. Schwartz. Mechanotransduction in vascular physiology and atherogenesis. Nat. Rev. Mol. Cell Biol. 10(1):53-62, 2009.

${ }^{24}$ Hazzard, T. M., and R. L. Stouffer. Angiogenesis in ovarian follicular and luteal development. Baillieres Best Pract. Res. Clin. Obstet. Gynaecol. 14(6):883-900, 2000.

${ }^{25}$ Holt, J. E., et al. CXCR4/SDF1 interaction inhibits the primordial to primary follicle transition in the neonatal mouse ovary. Dev. Biol. 293(2):449-460, 2006.

${ }^{26}$ Hornick, J., et al. Multiple follicle culture supports primary follicle growth through paracrine-acting signals. Reproduction 145(1):19-32, 2013.

${ }^{27}$ Hosaka, T., et al. Disruption of forkhead transcription factor (FOXO) family members in mice reveals their functional diversification. Proc. Natl. Acad. Sci. U.S.A. 101(9):2975-2980, 2004.

${ }^{28}$ Hsueh, A. J., et al. Hormonal regulation of the differentiation of cultured ovarian granulosa cells. Endocr. Rev. 5(1):76-127, 1984.

${ }^{29}$ Ishihara, S., et al. Substrate stiffness regulates temporary NF-kappaB activation via actomyosin contractions. Exp. Cell Res. 319(19):2916-2927, 2013.

${ }^{30}$ Karin, M., and A. Lin. NF-[kappa]B at the crossroads of life and death. Nat. Immunol. 3(3):221-227, 2002.

${ }^{31}$ Kastan, M. B., et al. A mammalian cell cycle checkpoint pathway utilizing p53 and GADD45 is defective in ataxiatelangiectasia. Cell 71(4):587-597, 1992.

${ }^{32}$ Kawamura, K., et al. Paracrine regulation of the resumption of oocyte meiosis by endothelin-1. Dev. Biol. 327(1):62-70, 2009.

${ }^{33}$ Kawasaki, F., et al. The clinical role of interleukin- 6 and interleukin-6 soluble receptor in human follicular fluids. Clin. Exp. Med. 3(1):27-31, 2003.

${ }^{34}$ Kim, D., J. Lee, and A. L. Johnson. Vascular endothelial growth factor and angiopoietins during hen ovarian follicle development. Gen. Comp. Endocrinol. 232:25-31, 2016.

${ }^{35}$ Knight, P. G., and C. Glister. TGF- $\beta$ superfamily members and ovarian follicle development. Reproduction 132(2):191206, 2006 
${ }^{36}$ Kreeger, P. K., et al. Regulation of mouse follicle development by follicle-stimulating hormone in a three-dimensional in vitro culture system is dependent on follicle stage and dose. Biol. Reprod. 73(5):942-950, 2005.

${ }^{37} \mathrm{Lau}, \mathrm{K}$. S., et al. In vivo systems analysis identifies spatial and temporal aspects of the modulation of TNF-alpha-induced apoptosis and proliferation by MAPKs. Sci. Signal 4(165):ra16, 2011.

${ }^{38}$ Lebbe, M., et al. The steroid metabolome in the isolated ovarian follicle and its response to androgen exposure and antagonism. Endocrinology 158(5):1474-1485, 2017.

${ }^{39}$ Liu, Z., et al. Interleukin-6: an autocrine regulator of the mouse cumulus cell-oocyte complex expansion process. Endocrinology 150(7):3360-3368, 2009.

${ }^{40}$ Maisonpierre, P. C., et al. Angiopoietin-2, a natural antagonist for Tie2 that disrupts in vivo angiogenesis. Science 277(5322):55-60, 1997.

${ }^{41}$ Makanji, Y., et al. Hypoxia-mediated carbohydrate metabolism and transport promote early-stage murine follicle growth and survival. Am J Physiol Endocrinol. Metab. 306(8):E893-E903, 2014.

${ }^{42} \mathrm{Man}, \mathrm{L}$., et al. Engineered endothelium provides angiogenic and paracrine stimulus to grafted human ovarian tissue. Sci. Rep. 7(1):8203, 2017.

${ }^{43}$ Mendez, U., H. Zhou, and A. Shikanov. Synthetic PEG Hydrogel for Engineering the Environment of Ovarian Follicles. In: Biomaterials for Tissue Engineering. Methods in Molecular Biology, Vol. 1758, edited by K. Chawla. New York: Humana Press, 2018, pp. 115-128.

${ }^{44}$ Molineux, G., et al. The effects on hematopoiesis of recombinant stem cell factor (ligand for c-kit) administered in vivo to mice either alone or in combination with granulocyte colony-stimulating factor. Blood 78(4):961-966, 1991.

${ }^{45}$ Motro, B., et al. Pattern of interleukin 6 gene expression in vivo suggests a role for this cytokine in angiogenesis. Proc Natl Acad Sci USA 87(8):3092-3096, 1990.

${ }^{46}$ Murray, S., et al. Regulation of granulosa cell-derived ovarian metalloproteinase inhibitor(s) by prolactin. J. Reprod. Fertil. 107(1):103-108, 1996.

${ }^{47}$ Nilsson, E., and M. K. Skinner. Cellular interactions that control primordial follicle development and folliculogenesis. J. Soc. Gynecol. Investig. 8:S17-S20, 2001.

${ }^{48}$ O'Dwyer, D. N., et al. The peripheral blood proteome signature of idiopathic pulmonary fibrosis is distinct from normal and is associated with novel immunological processes. Sci. Rep. 7:46560, 2017.

${ }^{49}$ Park, J.-Y., et al. EGF-like growth factors as mediators of LH action in the ovulatory follicle. Science 303(5658):682684, 2004.

${ }^{50}$ Penalver Bernabe, B., et al. Dynamic transcription factor activity networks in response to independently altered mechanical and adhesive microenvironmental cues. Integr. Biol. (Camb) 8(8):844-860, 2016.

${ }^{51}$ Sabbaghi, M., et al. IL-17A concentration of seminal plasma and follicular fluid in infertile men and women with various clinical diagnoses. Immunol. Invest. 43(7):617-626, 2014.

${ }^{52}$ Schneider, C. A., W. S. Rasband, and K. W. Eliceiri. NIH Image to ImageJ: 25 years of image analysis. Nat. Methods 9(7):671-675, 2012.

${ }^{53}$ Shikanov, A., et al. Interpenetrating fibrin-alginate matrices for in vitro ovarian follicle development. Biomaterials 30(29):5476-5485, 2009.
${ }^{54}$ Shikanov, A., et al. A method for ovarian follicle encapsulation and culture in a proteolytically degradable 3 dimensional system. J. Vis. Exp. 49:2695, 2011.

${ }^{55}$ Shikanov, A., et al. Hydrogel network design using multifunctional macromers to coordinate tissue maturation in ovarian follicle culture. Biomaterials 32(10):2524-2531, 2011.

${ }^{56}$ Shweiki, D., et al. Patterns of expression of vascular endothelial growth factor (VEGF) and VEGF receptors in mice suggest a role in hormonally regulated angiogenesis. $J$. Clin. Investig. 91(5):2235-2243, 1993.

${ }^{57}$ Skaznik-Wikiel, M. E., et al. Granulocyte colony-stimulating factor in conjunction with vascular endothelial growth factor maintains primordial follicle numbers in transplanted mouse ovaries. Fertil. Steril. 95(4):1405-1409, 2011.

${ }^{58}$ Smyth, G. K., et al. LIMMA: linear models for microarray data. In: Bioinformatics and Computational Biology Solutions Using $\mathrm{R}$ and Bioconductor, edited by $\mathrm{R}$. Gentleman, V. Carey, S. Dudoit, R. Irizarry, and W. Huber. New York: Springer, 2005, pp. 397-420.

${ }^{59}$ Srisuma, S., et al. Identification of genes promoting angiogenesis in mouse lung by transcriptional profiling. Am. J. Respir. Cell Mol. Biol. 29(2):172-179, 2003.

${ }^{60}$ Stouffer, R. L., et al. Regulation and action of angiogenic factors in the primate ovary. Arch. Med. Res. 32(6):567$575,2001$.

${ }^{61}$ Tagler, D., et al. Embryonic fibroblasts enable the culture of primary ovarian follicles within alginate hydrogels. Tissue Eng. Part A 18(11-12):1229-1238, 2012.

${ }^{62}$ Tamadon, A., et al. Efficient biomaterials for tissue engineering of female reproductive organs. Tissue Eng. Regener. Med. 13(5):447-454, 2016.

${ }^{63}$ Tamanini, C., and M. De Ambrogi. Angiogenesis in developing follicle and corpus luteum. Reprod. Domest. Anim. 39(4):206-216, 2004.

${ }^{64}$ Taniguchi, T. Cytokine signaling through nonreceptor protein tyrosine kinases. Science 268(5208):251-255, 1995.

${ }^{65}$ Tingen, C. M., et al. A macrophage and theca cell-enriched stromal cell population influences growth and survival of immature murine follicles in vitro. Reproduction 141(6):809-820, 2011.

${ }^{66}$ Trau, H. A., et al. Prostaglandin E2 and vascular endothelial growth factor A mediate angiogenesis of human ovarian follicular endothelial cells. Hum. Reprod. 31(2):436-444, 2016.

${ }^{67}$ van den Hurk, R., and J. Zhao. Formation of mammalian oocytes and their growth, differentiation and maturation within ovarian follicles. Theriogenology 63(6):1717-1751, 2005.

${ }^{68}$ Viedt, C., et al. Monocyte chemoattractant protein-1 induces proliferation and interleukin- 6 production in human smooth muscle cells by differential activation of nuclear factor-kappaB and activator protein-1. Arterioscler. Thromb. Vasc. Biol. 22(6):914-920, 2002.

${ }^{69}$ Wang, Y., S. Chan, and B. K. Tsang. Involvement of inhibitory nuclear factor-kappaB (NFkappaB)-independent NFkappaB activation in the gonadotropic regulation of $\mathrm{X}$ linked inhibitor of apoptosis expression during ovarian follicular development in vitro. Endocrinology 143(7):27322740, 2002.

${ }^{70}$ Weiss, M. S., et al. Dynamic, large-scale profiling of transcription factor activity from live cells in 3D culture. PLoS ONE 5(11):e14026, 2010. 
${ }^{71}$ Weiss, M. S., et al. Dynamic transcription factor activity and networks during ErbB2 breast oncogenesis and targeted therapy. Integr. Biol. (Camb) 6(12):1170-1182, 2014.

${ }^{72}$ West, E. R., et al. Physical properties of alginate hydrogels and their effects on in vitro follicle development. Biomaterials 28(30):4439-4448, 2007.

${ }^{73}$ Wigglesworth, K., et al. Transcriptomic diversification of developing cumulus and mural granulosa cells in mouse ovarian follicles. Biol. Reprod. 92(1):23, 2015.

${ }^{74} \mathrm{Xu}, \mathrm{M}$., et al. Tissue-engineered follicles produce live, fertile offspring. Tissue Eng. 12(10):2739-2746, 2006.

${ }^{75} \mathrm{Xu}$, M., et al. Encapsulated three-dimensional culture supports development of nonhuman primate secondary follicles. Biol. Reprod. 81(3):587-594, 2009.

${ }^{76} \mathrm{Xu}, \mathrm{M}$., et al. Secondary follicle growth and oocyte maturation by culture in alginate hydrogel following cryop- reservation of the ovary or individual follicles. Biotechnol. Bioeng. 103(2):378-386, 2009.

${ }^{77} \mathrm{Xu}$, J., et al. Secondary follicle growth and oocyte maturation during encapsulated three-dimensional culture in rhesus monkeys: effects of gonadotrophins, oxygen and fetuin. Hum. Reprod. 26(5):1061-1072, 2011.

${ }^{78}$ Yeung, F., et al. Modulation of NF- $\kappa \mathrm{B}-$ dependent transcription and cell survival by the SIRT1 deacetylase. EMBO J 23(12):2369-2380, 2004.

${ }^{79}$ Young, J. M., and A. S. McNeilly. Theca: the forgotten cell of the ovarian follicle. Reproduction 140(4):489-504, 2010.

${ }^{80}$ Zimmermann, R. C., et al. Vascular endothelial growth factor receptor 2-mediated angiogenesis is essential for gonadotropin-dependent follicle development. J. Clin. Investig. 112(5):659, 2003. 